(1)

CrossMark

\title{
Dual mechanism of action of T2 inhibitor therapies in virally induced exacerbations of asthma: evidence for a beneficial counter-regulation
}

\author{
John Efthimiou ${ }^{1}$, Chris Poll ${ }^{2}$ and Peter J. Barnes ${ }^{3}$ \\ Affiliations: ${ }^{1}$ Independent Respiratory Specialist, Oxford, UK. ${ }^{2}$ Independent Respiratory Scientist, Cambridge, \\ UK. ${ }^{3}$ National Heart and Lung Institute, Imperial College London, London, UK.
}

Correspondence: John Efthimiou, 5 Carey Close, Oxford, OX2 8HX, UK. E-mail: johnefthimiou1ahotmail.co.uk

@ERSpublications

T2 pathway inhibitors, including mAbs, significantly reduce asthma exacerbations, most of which are virus induced, and may act via a dual mechanism of action or counter-regulation, with targeted reduction in airway inflammation and enhanced IFN production http://ow.ly/N3zd30onQV9

Cite this article as: Efthimiou J, Poll C, Barnes PJ. Dual mechanism of action of T2 inhibitor therapies in virally induced exacerbations of asthma: evidence for a beneficial counter-regulation. Eur Respir J 2019; 54: 1802390 [https://doi.org/10.1183/13993003.02390-2018].

ABSTRACT Biological agents such as omalizumab and monoclonal antibodies (mAbs) that inhibit type 2 (T2) immunity significantly reduce exacerbations, which are mainly due to viral infections, when added to inhaled corticosteroids in patients with severe asthma. The mechanisms for the therapeutic benefit of T2 inhibitors in reducing virally induced exacerbations, however, remain to be fully elucidated. Pre-clinical and clinical evidence supports the existence of a close counter-regulation of the high-affinity IgE receptor and interferon (IFN) pathways, and a potential dual mechanism of action and therapeutic benefit for omalizumab and other T2 inhibitors that inhibit IgE activity, which may enhance the prevention and treatment of virally induced asthma exacerbations. Similar evidence regarding some novel T2 inhibitor therapies, including mAbs and small-molecule inhibitors, suggests that such a dual mechanism of action with enhancement of IFN production working through non-IgE pathways might also exist. The specific mechanisms for this dual effect could be related to the close counter-regulation between T2 and T1 immune pathways, and potential key underlying mechanisms are discussed. Further basic research and better understanding of these underlying counter-regulatory mechanisms could provide novel therapeutic targets for the prevention and treatment of virally induced asthma exacerbations, as well as T2- and non-T2-driven asthma. Future clinical research should examine the effects of T2 inhibitors on IFN responses and other T1 immune pathways, in addition to any effects on the frequency and severity of viral and other infections and related exacerbations in patients with asthma as a priority. 


\section{Introduction}

Respiratory viruses cause $\sim 80 \%$ of asthma exacerbations in children and adults $[1,2]$, with rhinoviruses (common cold viruses) being the most frequent, causing up to $65 \%$ of virally induced exacerbations [3-6]. Inhaled corticosteroids (ICSs) are known to improve asthma symptoms and decrease frequency of exacerbations, although virally induced exacerbations can occur even in well-controlled ICS-treated asthma [7]. Clearly, a component of virus-induced inflammation in asthma exacerbations is not controlled by regular ICS therapy [8] and there remains an unmet therapeutic need in these patients. Biological agents, such as omalizumab, an anti-IgE monoclonal antibody $(\mathrm{mAb})[9,10]$, and more recently other type 2 (T2) immune pathway blocking mAbs, such as mepolizumab and reslizumab (anti-interleukin (IL)-5 mAbs), benralizumab (anti-IL-5 receptor (R) $\alpha \mathrm{mAb}$ ), dupilumab (anti-IL-4R $\alpha \mathrm{mAb}$ ), and lebrikizumab (anti-IL-13 mAb) [11-21], have been developed for the treatment of patients with severe, particularly "T2-high" asthma (e.g. with raised IgE, eosinophils, exhaled nitric oxide fraction (FENO) and periostin) [22], and significantly reduce (i.e. prevent) exacerbations over and above that achieved with ICS. This suggests some complimentary efficacy of these biological agents and corticosteroids in preventing exacerbations overall, although the mechanisms of action, particularly with regard to virally induced exacerbations, remain to be fully explored.

\section{Search strategy and selection criteria}

References for this review were identified through searches of PubMed for articles published beginning in 1966 up to September 2018, by use of the terms T2 immunity, interferon (IFN), virus, asthma exacerbation, airway inflammation, T2-T1 counter-regulation and similar terms. Other relevant articles were identified through searches in Medscape and using the Google search engine. Articles of controlled studies, to help minimise bias, resulting from these searches and relevant references cited in those articles were reviewed. Articles published in or translated directly into English were included.

\section{Dual effects of anti-IgE on viral infections and asthma exacerbations}

A prospective randomised controlled field trial with omalizumab in children and adolescents with moderate to severe allergic asthma showed that omalizumab reduced the frequency and duration of viral upper respiratory tract infections, and reduced peak of viral shedding and related asthma exacerbations [23]. Another study in children and adolescents with moderate to severe asthma showed that omalizumab significantly reduced fall (autumn) exacerbation rates and also improved IFN- $\alpha$ response after rhinovirus infection ex vivo, and greater IFN- $\alpha$ increases were associated with fewer exacerbations [24]. Based on median IFN- $\alpha$ production in the omalizumab group, those subjects with IFN- $\alpha$ levels of less than the median had an approximately six-fold higher exacerbation rate compared with those who displayed a higher than median IFN- $\alpha$ response.

The underlying mechanism for this unexpected beneficial effect of omalizumab appears to be related to the fact that plasmacytoid dendritic cells, which produce large quantities of IFN- $\alpha$, express high-affinity IgE receptors (FcERI) on the cell surface. Virus-induced IFN- $\alpha$ responses are inversely related to expression of FceRI and are further inhibited by receptor cross-linking [25, 26]. These data suggest a negative counter-regulation of FcERI and IFN pathways in plasmacytoid dendritic cells, and that allergen-induced IgE cross-linking impairs plasmacytoid dendritic cell antiviral responses. Exactly how cross-linking of FceRI results in impaired IFN expression is unknown and the precise intracellular mechanism has not been described.

Omalizumab reduces surface expression of FceRI on plasmacytoid dendritic cells and blocks IgE binding to the receptor, thus enhancing IFN- $\alpha$ responses by reducing IgE receptor cross-linking by allergen [24]. Enhancing IFN generation limits viral replication and cell-to-cell spread of respiratory viruses, diminishing the extent of infection and severity of viral-related illness, including exacerbations in people with asthma (figure 1). The two aforementioned omalizumab studies provide direct clinical evidence that blocking $\operatorname{IgE}$ with omalizumab can improve viral clearance and reduce susceptibility to rhinovirus illness and related exacerbations of asthma [23, 24].

The importance of this dual mechanism of action is enhanced further by the observation that at least some patients with asthma, possibly if less well controlled, also have impaired IFN responses to rhinovirus in bronchial epithelial and other lung cells [27-30], so omalizumab may help to restore the antiviral immune response.

The clinical studies with omalizumab [23, 24] suggest dual mechanisms of action and therapeutic benefit, through both the specific mechanism of action of the drug (i.e. blocking circulating IgE and the related airway inflammatory response) and the enhancement of the innate immune response (i.e. increasing IFN), can come together in a complimentary manner to more effectively prevent virally induced asthma exacerbations. Such a dual effect may result from a beneficial counter-regulation following inhibition of 

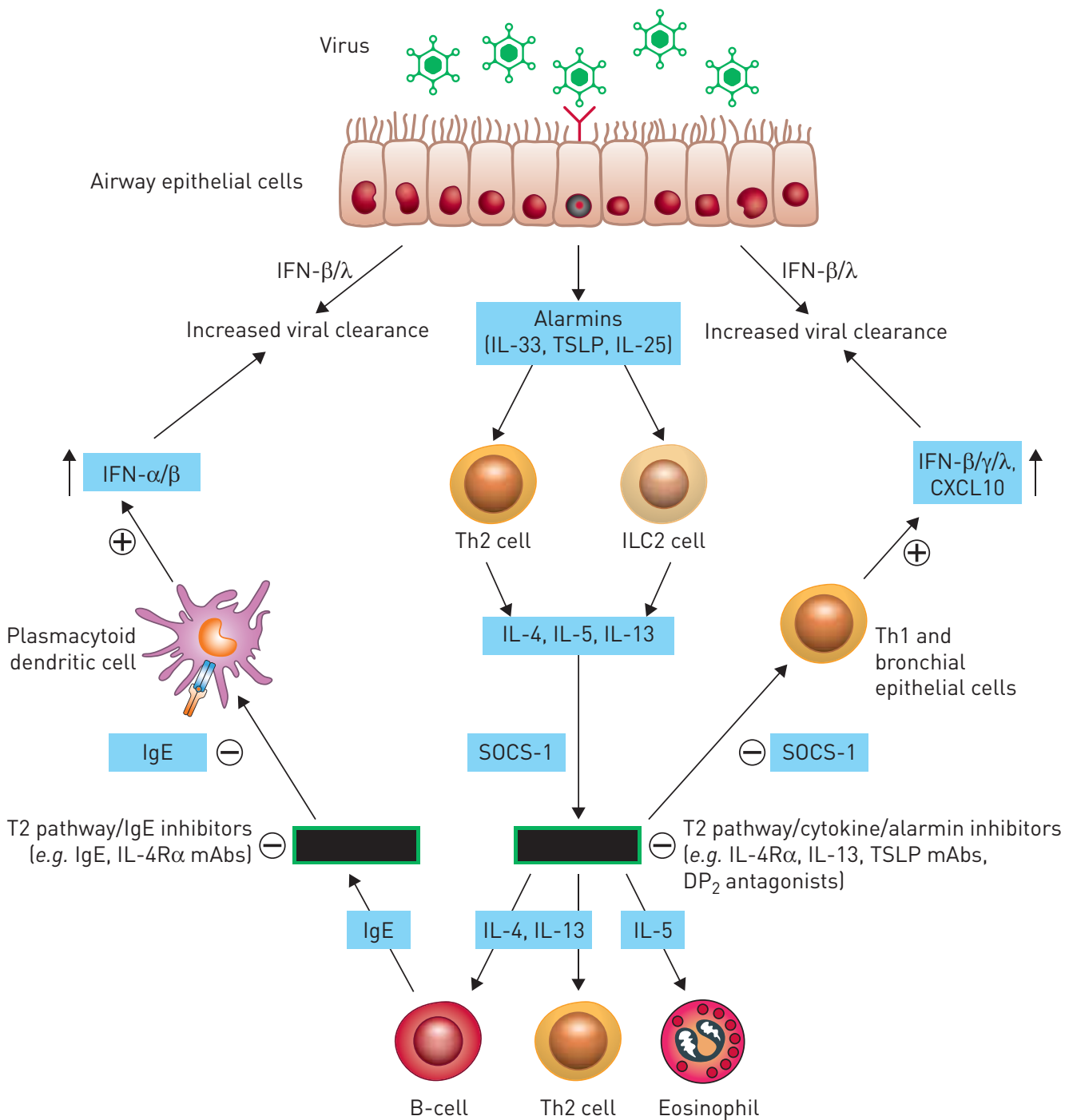

FIGURE 1 Examples of type 2 (T2) inhibitor therapy-induced T-helper type 1 (Th1) immune pathway counter-regulation associated with increased interferon (IFN) production, leading to potential dual mechanisms of action and clinical benefits in virally induced asthma exacerbations. IL: interleukin; R: receptor; TSLP: thymic stromal lymphopoietin; CXCL10: chemokine (C-X-C motif) ligand 10 (also known as IFN- $\gamma$-induced protein-10); ILC2: type 2 innate lymphoid cell; SOCS: suppressor of cytokine signalling; mAb: monoclonal antibody; $\mathrm{DP}_{2}$ : prostaglandin $\mathrm{D}_{2}$ receptor. Potential dual mechanisms of action and clinical benefits of T2 inhibitor therapies (e.g. anti-IgE, anti-IL-4R $\alpha$, anti-IL-13, anti-IL-33 and anti-TSLP mAbs) resulting from their combined T2 immune pathway inhibition and counter-regulated increase in IFN production helping to inhibit virally induced asthma exacerbations. Blocking IgE leads to increased type I IFN- $\alpha / \beta$ production from plasmacytoid dendritic cells, enhancing antiviral mechanisms. Blocking IL-4, IL-13, IL-33 or TSLP leads to increased type I IFN- $\beta$ and type III IFN- $\lambda$ production from, for example, Th1 and bronchial epithelial cells, broadly enhancing antiviral mechanisms. This secondary increase in IFN production is in addition to IFN- $\beta / \lambda$ produced by the bronchial epithelium in direct response to viral infection. Blocking either IgE or T2 cytokines therefore potentially inhibits virally induced asthma exacerbations through dual mechanisms of action. SOCS-1 is T2 cytokine inducible and a potent negative regulator of virus-induced IFN production; by reducing SOCS-1 levels, T2 inhibitors can increase IFN production from a number of key cells involved, and this may act as one of the pivotal mechanisms underlying the beneficial counter-regulation between $\mathrm{T} 2$ and $\mathrm{T} 1$ immune pathways relevant to virally induced asthma exacerbations.

this T2 immune pathway, resulting in an increase in part of the interconnected T1 immune pathway with increased IFN production [25].

\section{Dual effects of other T2 inhibitors on viral infections and asthma exacerbations}

In this review we refer to T2 responses as the general induction of cytokines IL-4, IL-5 and IL-13 and their associated biological effects, produced from either T-helper (Th) type 2 (Th2) T-lymphocytes or type 2 
innate lymphoid cells (ILC2). A Th2 response specifically refers to T-lymphocytes that have differentiated into Th2 cells and secrete IL-4, IL-5 and IL-13.

Complimentary, dual mechanisms of benefit may also apply to some of the novel T2 inhibitor therapies, including $\mathrm{mAbs}$ and small-molecule inhibitors, recently approved for clinical use or in clinical development for asthma, including anti-IL-5, anti-IL-4R $\alpha$, anti-IL-13 [11-20] and anti-alarmin (e.g. thymic stromal lymphopoietin (TSLP) and IL-33) [31,32] mAbs, and oral prostaglandin $\mathrm{D}_{2}$ receptor $\left(\mathrm{DP}_{2}\right)$ antagonists [33]. The potential complimentary mechanisms of action and clinical benefit with these novel T2 inhibitors clearly require further exploration, particularly through specific clinical studies in preventing and/or treating virally induced asthma exacerbations, in either field or experimental viral challenge settings. However, supportive pre-clinical and clinical data for similar dual beneficial effects with a number of T2 inhibitors already exist and are reviewed in the following sections.

\section{Interleukin-4}

IL-4 mediates important pro-inflammatory functions in asthma, including induction of the IgE isotype switching in B-cells, promotion of eosinophil transmigration across endothelium and differentiation of Th2 lymphocytes [34]. Both IL-4 and IL-13 can activate the type II IL-4R (IL-4R $\alpha /$ IL-13R $\alpha 1$ heterodimer) on a variety of cells, including epithelial cells, airway smooth muscle cells and fibroblasts [35]. In vitro studies in human bronchial epithelial cells show that IL-4 has a direct disruptive effect on airway epithelial barrier function [36]. Particularly relevant to this review, a negative association between bronchial epithelial IL-4 levels in asthmatic and atopic children and impaired IFN- $\beta$ and IFN- $\lambda$ responses from cultured human bronchial epithelial cells following rhinoviral infection has been described [37]. It has also been shown that human bronchial epithelial cells cultured with IL-4 are unable to mount a robust IFN response to rhinovirus infection and that IL-4 may be acting through inhibition of Toll-like receptor (TLR)-3 expression, impairing the immune response to viral infection [38]. The effects of IL-4 in vivo on both secondary immune responses by an adoptive transfer assay and primary immune responses by treatment of influenza virus-infected mice have demonstrated that IL-4 inhibits antiviral immunity [39]. The clearance of influenza virus from the lungs of infected BALB/c mice was significantly delayed after the transfer of virus-specific T-cells secondarily stimulated in the presence of IL-4. In addition, secondarily stimulated spleen cells from IL-4-treated mice contained significantly less IFN- $\gamma$ than did spleen cells from controls. More importantly, the treatment of mice with IL-4 resulted in a very significant delay in virus clearance. Thus, IL-4, at least in mice, can inhibit both primary and secondary antiviral immune responses. In addition, in another study in transgenic mice overexpressing IL-4, significantly delayed clearance of respiratory syncytial virus (RSV) from the lung was seen compared with control mice [40].

\section{Interleukin-13}

IL-13, along with other T2 cytokines, mediates important pro-inflammatory processes in asthma, including induction of the IgE isotype switching in B-cells, promotion of eosinophil trafficking into tissues, mucus hypersecretion and smooth muscle hypertrophy [35]. Although both IL-4 and IL-13 can activate the type II IL-4R (IL-4R $\alpha /$ IL-13R $\alpha 1$ heterodimer) on a variety of cells, including epithelial cells, airway smooth muscle cells and fibroblasts, IL-13, not IL-4, appears to mediate the main physiological features of asthma, including mucus hypersecretion, subepithelial fibrosis and airway hyperresponsiveness. IL-13 also appears to have a direct disruptive effect on airway epithelial barrier function in in vitro studies in human bronchial epithelial cells [36]. Higher levels of IL-13 have been shown to predispose to more severe viral infections, and to increase allergic airway inflammation and viral load in mice, with the latter correlating with suppressed IFN- $\beta$ and IFN- $\lambda$ responses. Blocking IL-13 reduced airway inflammation, hyperresponsiveness and viral load, and increased antiviral IFN responses [41]. These pre-clinical results suggest anti-IL-13 therapies may also have dual mechanisms of action relevant to virally induced asthma exacerbations.

Relevant here is the clinical demonstration that lebrikizumab, an anti-IL-13 mAb, significantly reduced the frequency of exacerbations in patients with moderate to severe asthma and raised blood periostin levels in two pooled phase $2 \mathrm{~b}$ studies [16], most of these exacerbations typically being caused by viral infections, as discussed earlier [1, 2]. Lebrikizumab was also shown to significantly reduce exacerbations in biomarker-high patients with uncontrolled asthma in one of its two replicate phase 3 studies LAVOLTA I, although the decrease in exacerbations was not significant in LAVOLTA II [42]. However, despite the lack of consistent significant efficacy of lebrikizumab in reducing asthma exacerbations in its phase 3 studies, it can be said that significant efficacy in reducing exacerbations was seen in two large randomised controlled phase $2 \mathrm{~b}$ and 3 trials, at least with this anti-IL-13 mAb. Another anti-IL-13 mAb, tralokinumab, also demonstrated a significant reduction in exacerbation rate in patients with severe asthma and high FENO levels in one phase 3 trial (STRATOS 1), but failed to do so in the second phase 3 study in a similar population (STRATOS 2) [43]. Overall these clinical data suggest that targeting IL-13 alone may not be 
sufficiently central to the pathophysiology to consistently prevent asthma exacerbations, despite efficacy being seen in several phase $2 \mathrm{~b}$ and 3 studies, in biomarker-high, severe asthma patients.

\section{Interleukin-4 receptor $\alpha$}

IL-4 and IL-13 clearly play important and additive roles in the initiation and propagation of the airway inflammation associated with allergy and viral infection. Blocking either of these T2 cytokines can reduce airway inflammation, hyperresponsiveness and viral load, and increase antiviral IFN responses, at least in mice [37-41]. It seems possible therefore that blocking both these cytokines will result in additive anti-inflammatory and antiviral effects, including reduced airway inflammation and increased IFN responses, potentially resulting in dual clinical benefits with regard to reducing virally induced asthma exacerbations.

IL-4 and IL-13 share many biological functions due to their ability to signal through a unique network of common receptors. IL-4 can signal through the type I IL-4R (IL-4R $\alpha$ heterodimer) and the type II IL-4R (IL-4R $\alpha / \mathrm{IL}-13 \mathrm{R} \alpha 1$ heterodimer), but IL-13 only signals through the type II IL-4R [44, 45]. Consequently IL-4 and IL-13 cytokine responses can be completely abrogated on cells lacking IL-4R $\alpha$ expression.

The infection of gene knockout mice and wild-type mice with vaccinia virus shows that IL-4, IL-13, and signal transducer and activator of transcription (STAT)-6 are required to increase IL-4R $\alpha$ expression on $\mathrm{CD}^{+}$T-cells, but not to increase IFN- $\gamma$ [46]. STAT-6-dependent elevation of IL-4R $\alpha$ expression on CD8 ${ }^{+}$ $\mathrm{T}$-cells was a feature of poor quality antiviral $\mathrm{CD}^{+} \mathrm{T}$-cell immunity as measured by the production of IFN- $\gamma$ and tumour necrosis factor (TNF)- $\alpha$ in response to vaccinia virus stimulation in vitro. Downregulation of IL-4R $\alpha$ appears to be a mechanism by which $\mathrm{CD} 8^{+} \mathrm{T}$-cells reduce responsiveness to IL-4 and IL-13, which can improve the quality of antiviral $\mathrm{CD}^{+} \mathrm{T}$-cell immunity. The mechanisms by which IL-4- and/or IL-13-mediated increases in IL-4R $\alpha$ expression on $\mathrm{CD}^{+} \mathrm{T}$-cells lead to reduced IFN- $\gamma$ and TNF- $\alpha$ production are unclear. However, one potential mechanism that may be involved is the activation of suppressor of cytokine signalling (SOCS)- 1 and SOCS-3 on antiviral CD8 ${ }^{+}$T-cells, as IL-4and IL-13-induced activation of these inhibitory proteins has previously been reported to attenuate IFN- $\gamma$ and TNF- $\alpha$ production in keratinocytes [47].

Additionally, it is well known that both IL-4 and IL-13 mediate induction of IgE isotype switching in B-cells, increasing IgE production and hence decreasing IFN release from plasmacytoid dendritic cells [34, $35,48]$. Inhibiting IL-4 and IL-13 with an IL-4R $\alpha$ inhibitor therefore may inhibit SOCS-1/3 and IgE, leading to an increase in IFN production through both these mechanisms (figure 1).

Relevant here, dupilumab, a novel, fully human anti-IL-4R $\alpha \mathrm{mAb}$, recently approved in the USA for the treatment of moderate and severe eosinophilic asthma patients [49], inhibits both IL-4 and IL-13 signalling, key drivers of T2-mediated inflammation in asthma. In a phase $2 \mathrm{~b}$ study dupilumab significantly reduced exacerbations in patients with moderate to severe asthma, by up to $81 \%$ with and up to $68 \%$ without raised blood eosinophils [18]. More recently, the results of a large phase 3 study (LIBERTY ASTHMA QUEST) demonstrated that dupilumab significantly reduced the rate of severe exacerbations by up to $66 \%$ in patients with raised blood eosinophils, on top of ICSs, in patients with moderate to severe asthma, in addition to significant benefits on lung function, asthma control and asthma-specific quality of life [20].

The broad range of clinical benefits seen also raises the possibility that targeting both IL-4 and IL-13 may have important additive effects on the underlying pathophysiology, as well as on the associated clinical manifestations of asthma. In addition, in the phase 3 asthma study, the rates of viral upper respiratory tract infections, upper respiratory tract infections and bronchitis were all overall numerically lower, although not statistically, in the dupilumab group compared with placebo, consistent with a potential antiviral infection effect [20]. Moreover, a meta-analysis of eight randomised controlled trials with dupilumab in atopic dermatitis, another T2 inflammatory disorder, demonstrated a significantly lower frequency of skin infections and of eczema herpeticum versus placebo, again consistent with a potential antiviral infection effect, in addition to its expected T2 inhibitor anti-inflammatory efficacy [50].

\section{Interleukin-5}

IL-5 is the main cytokine involved in the production, trafficking and regulation of eosinophils, which are thought to play a key role in the pathophysiology of asthma and other allergic disorders [51]. Eosinophils participate in the induction of airway hyperresponsiveness, mucus hypersecretion, airway epithelial cell damage and remodelling, i.e. characteristic features of asthma, and are also thought to be important for innate and adaptive immune responses to various pathogens [52-54]. Eosinophils are known to be involved in clearing parasitic infections, and have been implicated in the host response to respiratory viral infections in pre-clinical models of RSV [55] and parainfluenza virus infections [56, 57]. In vitro 
eosinophils co-cultured with lung epithelial cells have been shown to reduce RSV infectivity in lung epithelial cells and in vivo to promote clearance of RSV in a mouse model [55]. In contrast to wild-type mice, RSV clearance from lung tissue was more rapid in hyper-eosinophilic (IL-5 transgenic) mice. Hyper-eosinophilia in the airways of infected mice also correlated with increased expression of IFN regulatory factor (IRF)-7 and IFN- $\beta$. Eosinophil activation proteins may be key antiviral mechanisms, and it has been shown that eosinophil cationic protein [58] and eosinophil-derived neurotoxin [59] are RNases with direct antiviral activity and can enter viral capsids to degrade RNA in RSV. Human and mouse eosinophils have been demonstrated to exert antiviral activity against parainfluenza virus by nitric oxide production [56]. Collectively, these results demonstrate that eosinophils can protect against respiratory viral infections and appear to promote virus clearance, at least in mice in vivo.

In contrast, IL-5-induced airway eosinophilia reduces TLR-7 expression and inhibits antiviral IFN responses to rhinovirus, resulting in exaggerated inflammatory responses [60]. In allergic TLR-7-deficient $\left(T l r 7^{-l-}\right)$ mice, rhinovirus exposure resulted in impaired production of IFN compared with wild-type controls, with higher rhinovirus replication in the lower airways and exaggerated eosinophilic inflammation. In addition, in bronchial biopsies from moderate to severe asthma subjects with eosinophilic airway inflammation, TLR-7 and IFN- $\lambda 2 / 3$ expression levels were reduced [60]. Similarly rhinovirus-induced IFN production in bronchial epithelial cells was inversely related to eosinophil counts in biopsies from pre-school children with mild asthma [37]. This implicates IL-5-induced airways eosinophilia as a negative regulator of TLR-7 expression and antiviral IFN responses, and provides a molecular mechanism underpinning the effect of eosinophil-targeted treatments for the prevention of virally induced asthma exacerbations.

In humans, eosinophil and IL-5 levels have been reported to increase in the airways of asthma subjects following experimental rhinovirus infection [61], and both higher baseline blood eosinophil [62] and airway IL-5 levels have also been associated with greater disease severity following viral infection in this model [63]. The anti-IL-5 mAbs approved during the last 3 years for patients with severe asthma, such as mepolizumab, reslizumab and benralizumab, appear to significantly reduce all types of exacerbations when added to ICSs [11-15]. The fact that respiratory viruses are the commonest cause of asthma exacerbations, combined with the observation that biologicals targeting the IL-5 pathways significantly decrease eosinophilic inflammation and frequency of exacerbations, argues for at least some potential role of eosinophils in virally induced exacerbations.

Relevant here, in a recent experimental rhinovirus challenge study in subjects with mild asthma, mepolizumab showed no significant effect on the fall in lung function or loss of asthma control post-viral infection and produced mixed immunological changes, including potentially both antiviral and proviral effects, as well as a significant increase in viral load, although the latter was only measured at one time-point [64]. One explanation provided was that mepolizumab reduced eosinophil numbers but did not prevent activation of remaining eosinophils in response to rhinovirus challenge. Although the latter explanation may have been partly responsible in this specific mild asthma population, these results are also consistent with an ambivalent or conflicted immunological role of eosinophils with regard to viral infections. It seems likely in this context that besides any direct anti-inflammatory effects, IL-5 and eosinophil inhibition can lead to a number of both proviral and antiviral immune effects, and that the final clinical and virological result will therefore be determined by the net balance of these opposing effects and also potentially on the specific patient phenotype and endotype (i.e. underlying disease mechanism) involved. Given the ambiguity of the pre-clinical and clinical data in this regard, it would be helpful to conduct further suitably designed field and viral challenge clinical studies, in well-defined asthma populations, specifically evaluating the effects of anti-IL-5 mAbs, in preventing and treating virally induced asthma exacerbations, including the key underlying inflammatory and antiviral immune responses potentially impacted.

\section{Interleukin-33}

IL-33, produced by the airway epithelial cells on activation by allergens or viruses, is one of the key alarmins and upstream drivers of T2-mediated inflammation [65]. As an upstream diver of T2 inflammation, IL-33, like the other alarmins TSLP and IL-25, can impact a broad range of key cells, including T2 cells, epithelial cells, airway smooth muscle cells and fibroblasts, potentially mediating most of the key physiological features of asthma, including mucus hypersecretion, subepithelial fibrosis and airway hyperresponsiveness. Compared with individual T2 cytokines (e.g. IL-4, IL-5 and IL-13), in the overall hierarchy of the asthma inflammatory process, IL-33 and the other alarmins are more upstream and therefore anticipated to have at least as potent or possibly even greater clinical effects. Regarding viral infections, IL-33 has also been shown to play an important role in an experimental model of rhinovirus-induced exacerbations in people with asthma, and IL-33 induction correlates with viral load 
and exacerbation severity [61]. More recently, in a mouse model of allergen- and rhinovirus-induced asthma airway inflammation, it has been shown that IL-33 drives T2 inflammation and that anti-IL-33 treatment not only dampens T2 inflammation but also boosts antiviral cytokines and immunity and accelerates rhinovirus clearance [66]. Similarly, in a mouse model of allergen- and influenza-induced airway inflammation, blocking the IL-33 receptor (ST2) with a mAb dramatically reduced eosinophils, neutrophils, dendritic cells, B- and T-cells, and ILC2 cells in the airway, as well as increasing lung T1 immune pathway cytokines and reducing viral replication [67]. These data suggest that blocking IL-33 not only reduces virally induced T2 cytokines and airway inflammation, but also indirectly enhances antiviral immunity and viral clearance, and supports a potential dual mechanism of action for reducing virally induced asthma airway inflammation and exacerbations [68]. Several anti-IL-33 antibodies are now in clinical development [69-71] and clinical data demonstrating their anticipated effect in reducing asthma exacerbations, and ideally of their effect on antiviral immunity, through blocking IL-33, are therefore eagerly awaited. However, as with individual T2 cytokines, blocking individual alarmins with highly specific mAbs may still allow for escape mechanisms to step in and to reduce any potential clinical benefits, albeit to a lesser extent on account of their broader mechanisms of action [66, 67], and only the relevant clinical data with specific alarmin inhibitors will help address this question.

\section{Thymic stromal lymphopoietin}

Another alarmin produced by airway epithelial cells, TSLP, may also play an important upstream role in initiating inflammation triggered in response to viruses and allergens in asthma. TSLP is significantly upregulated by the TLR-3 ligand (double-stranded (ds) RNA) and similarly by rhinovirus infection, and is also synergistically enhanced by a combination of IL-4 and dsRNA in bronchial epithelial cells in vitro [72]. The induction of TSLP by dsRNA is dependent upon NF- $\kappa B$ and IRF-3 signalling via TLR-3. This dependency implies that respiratory viral infection and the recruitment of T2 cytokine-producing cells may amplify T2 inflammation via the induction of TSLP in the asthmatic airway.

Nasal epithelial cells, largely from patients who are atopic with nasal polyps, release TSLP when stimulated by polyinosinic-polycytidylic (poly(I:C)) acid (a TLR-3 activator), suggesting that viral infection may contribute to and amplify the T2 immune response through TSLP production [73]. Primary bronchial epithelial cells from subjects with asthma show an exaggerated secretion of TSLP after dsRNA exposure [74] and naturally occurring rhinovirus-induced asthma exacerbations have been associated with in vivo airway secretion of TSLP [75].

In vivo, in a mouse model with a baseline of allergic asthma inflammation, pulmonary TSLP was induced at exacerbations exclusively evoked by rhinovirus infection or poly(I:C) [76]. In mice, RSV infection induces an increase in the number of IL-13-producing ILC2 cells, with a concurrent increase in total lung IL-13 and TSLP levels [77]. TSLP receptor knockout mice failed to mount an IL-13-producing ILC2 cell response to RSV infection, and showed decreased airway mucus and reactivity compared with wild-type mice. Additionally, neutralisation of TSLP significantly attenuated the RSV-induced IL-13-producing ILC2 cell response. These data demonstrate that RSV induces IL-13-producing ILC2 cell proliferation and activation through a TSLP-dependent mechanism that is likely to worsen asthma inflammation, at least in this murine model.

Indeed, tezepelumab, a fully human anti-TSLP mAb, has already been tested in a phase $2 \mathrm{~b}$ trial in patients with moderate to severe asthma and demonstrated a significant reduction in asthma exacerbations over 52 weeks when used in addition to regular ICS, and unlike some of the other T2 inhibitor therapies, promisingly this effect was independent of the baseline level of eosinophils [31]. The effect of this anti-TSLP mAb specifically on virally induced exacerbations, viral infections and the related immune responses, however, remains to be explored. The finding that respiratory viral infection may amplify T2 inflammation via the induction of TSLP in the asthmatic airway suggests that anti-TSLP mAbs may also block some of the key T2 inflammatory effects associated with respiratory viral infection and at least through this mechanism help to prevent virally induced asthma exacerbations.

\section{Prostaglandin $D_{2}$}

Regarding $\mathrm{DP}_{2}$ receptor antagonists (previously known as CRTH2 (chemoattractant receptor-homologous molecule expressed on Th2 cells) antagonists), a class of small-molecule T2 inhibitors, fevipiprant, an oral agent, has demonstrated novel anti-inflammatory effects on blood and airway gene expression in patients with persistent eosinophilic asthma, including downregulation of genes associated with Th2 humoral immunity and upregulation of genes linked to Th1/Th17 biology [78]. These data support a reciprocal regulation of T2-related genes and T1-related genes post-treatment with fevipiprant, consistent with the counter-regulation hypothesis proposed with the biological T2 inhibitors described earlier. However, specific data on IFN genes and their related biological responses with $\mathrm{DP}_{2}$ antagonists are awaited. 
With regard to clinical data, fevipiprant, currently in phase 3 clinical development for treatment of uncontrolled severe asthma, has also been shown to significantly improve lung function in patients with moderate asthma in a phase $2 \mathrm{~b}$ study [33] and the results on reducing exacerbations from the ongoing phase 3 programme are eagerly anticipated [79]. Of direct clinical relevance to the potential dual mechanism of clinical benefit of such $\mathrm{T} 2$ inhibitors, another oral $\mathrm{DP}_{2}$ antagonist, timapiprant (OC000459), has been associated with a significantly reduced frequency of respiratory tract infections, as well as improvements in lung function and asthma-related quality of life compared with placebo in a phase 2 trial in patients with moderate asthma [80]. Moreover, the results of an ongoing study in the human rhinovirus-induced asthma exacerbation model with timapiprant may help to better define the detailed clinical effects and underlying mechanisms of benefit in virally induced exacerbations [81].

In addition to the aforementioned omalizumab $[13,14]$ and dupilumab studies $[20,44]$ demonstrating a reduction in viral and possibly other respiratory infections, the results of the timapiprant study in asthma [74] provides further clinical support for a potential dual mechanism of action and therapeutic benefit of another class of T2 inhibitor of direct relevance to virally induced asthma exacerbations. These clinical data (table 1) also suggest that a review of the rates of all respiratory infections, and particularly viral infections and related exacerbations, ideally with assessment of IFN production, in already completed and ongoing clinical trials in asthma, as well as more robust prospective investigation in future phase 2 and 3 clinical trials, with all T2 inhibitor therapies, is warranted.

TABLE 1 Summary of type 2 inhibitor therapy effects on asthma exacerbations, respiratory infections and interferon (IFN) production

\begin{tabular}{|c|c|c|}
\hline Target & Drug & Main findings [ref.] \\
\hline \multirow[t]{4}{*}{$\lg E$} & \multirow[t]{4}{*}{ Omalizumab } & Significant reduction in exacerbation rate in adults with asthma by $26 \%$ [9] \\
\hline & & Significant reduction in exacerbation rate in children with asthma by $38 \%$ [10] \\
\hline & & $\begin{array}{l}\text { Significantly reduced exacerbations and restored IFN- } \alpha \text { responses to rhinovirus in peripheral blood } \\
\text { mononuclear cells in children/adolescents with asthma [24] }\end{array}$ \\
\hline & & $\begin{array}{l}\text { Reduced exacerbation frequency, frequency of rhinovirus illness, duration of rhinovirus infection and peak } \\
\text { rhinovirus shedding in children/adolescents with asthma [23] }\end{array}$ \\
\hline IL-5 & Mepolizumab & $\begin{array}{l}\text { Significant reduction in clinically significant exacerbation rates of up to } 52 \% \text { in a phase } 3 \text { study in patients with } \\
\text { eosinophilic asthma (DREAM); no obvious effect on viral infections seen in reported adverse events in a phase } \\
3 \text { study (but not specifically tested) [12]; no significant benefit on clinical end-points or viral load in } \\
\text { rhinovirus-induced asthma exacerbations using an experimental viral challenge model (MATERIAL study) [64] }\end{array}$ \\
\hline IL-5R $\alpha$ & Benralizumab & $\begin{array}{l}\text { Significant reduction in annualised exacerbation rates of up to } 51 \% \text { in two phase } 3 \text { studies in patients with } \\
\text { eosinophilic asthma (SIROCCO and CALIMA); no obvious effect on viral infections seen in reported adverse } \\
\text { events in phase } 3 \text { studies (but not specifically tested) }[14,15]\end{array}$ \\
\hline IL-5 & Reslizumab & $\begin{array}{l}\text { Significant reduction in annualised exacerbation rates of up to } 59 \% \text { in two replicate phase } 3 \text { studies in patients } \\
\text { with eosinophilic asthma; no obvious effect on viral infections seen in reported adverse events in phase } 3 \\
\text { studies (but not specifically tested) [13] }\end{array}$ \\
\hline IL-13 & Lebrikizumab & $\begin{array}{l}\text { Significant reduction in annualised exacerbation rate of up to } 51 \% \text { in biomarker-high patients in one phase } 3 \\
\text { study (LAVOLTA I), but not significant (up to 36\%) in a replicate second phase } 3 \text { study (LAVOLTA II); no obvious } \\
\text { effect on viral infections seen in reported adverse events in phase } 3 \text { studies (but not specifically tested) [42] }\end{array}$ \\
\hline IL-13 & Tralokinumab & $\begin{array}{l}\text { Significant reduction in annualised exacerbation rate of up to } 44 \% \text { in patients with high FENO in one phase } 3 \\
\text { study (STRATOS 1), but not significant (up to } 16 \% \text { ) in patients with high FENO in a second phase } 3 \text { study } \\
\text { (STRATOS 2); no obvious effect on viral infections seen in reported adverse events in phase } 3 \text { studies (but not } \\
\text { specifically tested) [43] }\end{array}$ \\
\hline IL-4R $\alpha$ & Dupilumab & $\begin{array}{l}\text { Significant reduction in annualised exacerbation rate of up to } 48 \% \text { in all patients with moderate to severe } \\
\text { asthma and up to } 66 \% \text { in patients with raised blood eosinophils in a phase } 3 \text { study (LIBERTY ASTHMA } \\
\text { QUEST); trend for lower frequencies of viral and upper respiratory tract infections, influenza, and bronchitis in } \\
\text { dupilumab versus placebo seen in reported adverse events in a phase } 3 \text { study (but not specifically tested) [20] }\end{array}$ \\
\hline TSLP & Tezepilumab & $\begin{array}{l}\text { Significant reduction in annualised exacerbation rate of up to } 71 \% \% \text { in patients with moderate to severe } \\
\text { asthma, regardless of blood eosinophil count, in a phase } 2 \text { b study; no obvious effect on viral infections seen } \\
\text { in reported adverse events in a phase } 2 \text { b study (but not specifically tested) [31] }\end{array}$ \\
\hline $\begin{array}{l}\text { DP }_{2} \\
\text { (CRTH2) }\end{array}$ & Fevipiprant & $\begin{array}{l}\text { Significant increase in FEV1 in patients with atopic uncontrolled asthma patients in a phase } 2 \text { b study; phase } 3 \\
\text { results and efficacy on exacerbations awaited; no obvious effect on viral infections seen in reported adverse } \\
\text { events in a phase } 2 \text { b study (but not specifically tested) [33] }\end{array}$ \\
\hline & Tamipiprant & $\begin{array}{l}\text { Significantly improvements in FEV1 and quality of life and significant reduction in frequency of respiratory tract } \\
\text { infections seen in reported adverse events in a phase } 2 \text { trial in patients with moderate asthma [80] }\end{array}$ \\
\hline
\end{tabular}

IL: interleukin; R: receptor; TSLP: thymic stromal lymphopoietin; $\mathrm{DP}_{2}$ : prostaglandin $\mathrm{D}_{2}$ receptor; CRTH2: chemoattractant receptorhomologous molecule expressed on T-helper type 2 cells; FENo: exhaled nitric oxide fraction; FEV1: forced expiratory volume in 1 s. 


\section{Summary of T2 inhibitor efficacy on asthma exacerbations}

The T2 inhibitors we described are those that have reached at least phase 1 in clinical development, and mostly have already demonstrated a significant reduction in overall asthma exacerbations rates and/or lung function (table 1). Although it is not possible to be conclusive without specific data on exacerbation aetiology, as it is known that most of these are virally induced [1-6], it is certainly conceivable that these agents are also at least partly effective at reducing virally induced exacerbations. Whether the reduction in exacerbation frequency seen in these studies is due to a reduction in viral infection associated with enhancement of the related immune response or that viral infection is unaffected but the consequent inflammatory response is diminished is uncertain. However, in the aforementioned omalizumab studies, viral illness and viral load, in addition to asthma exacerbations, were significantly reduced. Moreover, rhinovirus-induced IFN production was increased, which was itself associated with lower exacerbation frequency, in the omalizumab-treated group, supporting the dual mechanism of action and hypothesis proposed here $[23,24]$. Ideally, therefore, all T2 inhibitor therapies should also be tested on their potential to inhibit virally induced respiratory illness and to enhance antiviral IFN responses, as well to reduce asthma exacerbations. This could be undertaken in field studies, as was carried out with omalizumab, or in the rhinovirus challenge setting, where a few T2 inhibitors have been [64] or are currently being [81] tested.

\section{Virally induced exacerbations in severe asthma}

Regarding patients with severe asthma, in whom exacerbations can be frequent and severe [82, 83], and for whom most of the $\mathrm{T} 2$ inhibitor mAbs are primarily indicated, whether the majority of exacerbations are due to viruses remains uncertain. However, the overall evidence supporting viruses causing most asthma exacerbations in children and adults is strong and consistent $[1-6,84]$, and it seems likely that this will also apply at least to some extent in severe asthma.

Patients with severe asthma and frequent exacerbations appear to experience these throughout the year, and besides respiratory viral infections, it is plausible that poor control of airway inflammation is one of the underlying and main causes. However, it is recognised that severe asthma represents a complex group of patients in whom a number of different factors may contribute to their exacerbations (e.g. viral infection, allergens, poor controller therapy efficacy and/or adherence, and other medical problems including gastro-oesophageal reflux, obesity, psychosocial factors and other respiratory disorders) [85]. In addition, frequent exacerbations do not just occur in severe asthma and indeed the large, prospective, observational TENOR study found that the risk of recurrent exacerbations in adolescents and adults was independent of asthma severity, but strongly predicted by recent exacerbations [86].

It is also not possible to reliably predict the occurrence of virally induced exacerbations simply on a seasonal basis, as respiratory viruses do not all cause exacerbations during just one period of the year, and rates of hospitalisation and deaths for asthma have been shown to be similar across seasons [87]. Relevant here, a number of different respiratory viruses can cause asthma exacerbations, including rhinovirus, influenza, coronavirus, parainfluenza and RSV, with most of these having a different seasonal propensity $[3,4,6,88]$. For example, exacerbations precipitated by rhinovirus can be documented throughout the year, with a predilection for late spring and autumn months, whereas influenza tends to cause exacerbations in the winter months [89].

Also relevant here, inhaled IFN- $\beta$ given to patients with asthma, allowed to continue use of ICS, at the start of a common cold did not reduce the consequent asthma worsening overall [90]. An exploratory subgroup analysis, however, showed a significant improvement in asthma worsening in patients with more severe asthma. A further trial with inhaled IFN- $\beta$ in patients with moderate to severe asthma aiming to reduce the frequency of common cold-induced severe exacerbations was, however, stopped early due to a low severe exacerbation event rate that compromised the aims of the study [91]. Moreover, the apparent lack of efficacy of giving exogenous IFN alone in virally induced asthma exacerbations may not be surprising, unless the subjects selected have a substantial deficiency in their endogenous IFN response, besides any potential limitation related to inhaled IFN sufficiently reaching the right target lung cells. Additionally, we hypothesise giving exogenous IFN alone, without a T2 inhibitor or adequate corticosteroid therapy, is less likely to have a significant effect in virally induced asthma exacerbations, as only one of the two key mechanisms involved is improved by this approach, and both may be necessary to effectively prevent and treat such exacerbations.

Many exacerbations seen in severe asthma could be caused by viruses, although this appears to be a relative gap in our knowledge and does not appear to have been specifically explored in any prospective or retrospective clinical trials evaluating the aetiology of exacerbations with definitive virology, and such studies would clearly be of value. This data would also help to evaluate and place in a more complete perspective the current hypothesis of a potential dual mechanism of action and clinical benefit of the newer T2 inhibitors in virally induced exacerbations in patients with severe asthma. 


\section{Molecular mechanisms of T2-T1 counter-regulation}

The underlying mechanisms for the potential dual effects of T2 pathway inhibition and associated antiviral immune response enhancement may be related to the close negative regulation or counter-regulation existing between T2 and T1 immune pathways [92, 93].

A T1 to T2 switching is well recognised in the pathogenesis of asthma, with a relative increase in T2 responses in combination with a decrease in $\mathrm{T} 1$ responses, thought to arise at least in part due to a relative lack in stimuli of the T1 system, such as infection and endotoxin exposure, early in life [94]. Evidence for such a shift in the Th1 to Th2 balance derives from studies of asthma in cellular and murine models [95], and from human studies profiling cytokine production [96]. In addition, atopic subjects are thought to respond to environmental allergens with an increased synthesis of Th2 cytokines and IgE, together with a decrease in Th1 cytokines, although the precise mechanism for this Th1 to Th2 switch is not clear [97]. A possible mechanism to help explain the latter is suggested by at least one key T2 cytokine, IL-4, which encourages the differentiation of Th2 cells and inhibits the development of Th1 cells $[95,97,98]$. Indeed, T1 to T2 switching, associated with T1 cell activity declining as T2 cell activity increases, is also well recognised in a number of other viral infections [92].

In addition, blocking T2 pathways in vitro and in vivo results in an increase in T1 pathway activity, and hence potentially to increased antiviral IFN responses, which are predominantly T1 driven [68, 99]. Substantial evidence also specifically demonstrates that T2 pathways and type I and III IFNs can exhibit a similar counter-regulation on the expression or action of each other [99]. This includes that described earlier between high-affinity IgE receptors and IFN pathways in plasmacytoid dendritic cells and how allergen-induced IgE cross-linking impairs plasmacytoid dendritic cell antiviral responses (figure 1) $[25,26]$. Impaired IFN- $\lambda$ production in cultured asthmatic primary bronchial epithelial cells negatively correlates with sputum eosinophilia [28], and both serum IgE and IL-4 immunoreactivity in bronchial biopsy specimens [37]. IFN- $\alpha$ or TLR-7 agonists can suppress Th2 cell polarisation in pure T-cell and mixed leukocyte culture systems, downregulating levels of GATA3 transcription factor, IL-4, IL-13 and IL-5 [100, 101]. IFN- $\alpha$ also downregulates FceRI $\alpha$ mRNA levels on peripheral blood mononuclear cells, highlighting a counter-regulatory capacity for IFN- $\alpha$ on FceRI expression [102].

Human ILC2 cells cultured with IL-33 and IL-17 produce the T2 cytokines IL-4, IL-5, IL-13 and IL-9, which are downregulated in the presence of IFN- $\beta$ [103]. This latter study is the first to show that, in a similar manner to Th2 cells, innate lymphoid cells are also affected by IFNs and that their T2 function might be increased in an environment that lacks IFN. In human bronchial epithelial cells, pre-treatment with IL-4 or IL-13 reduces rhinovirus-induced IFN- $\beta$ and/or IFN- $\lambda$ levels; this suppression is specific for IFNs and does not inhibit pro-inflammatory cytokines, such as the chemokine (C-X-C motif) ligand CXCL8 [38]. Additionally, other non-T2 cytokines, such as IL-2, do not have this inhibitory effect on IFNs, again highlighting the unique relationship between T2 cytokines and the innate IFN response.

In vivo support for this counter-regulation is also derived from at least one mouse study, in which IFN- $\lambda$ administered through the airways suppressed the generation of allergic airways inflammation in an ovalbumin sensitisation and challenge model that is Th2 driven. This mechanism involves downregulation of OX40 ligand on dendritic cells, leading to less Th2 cell polarisation [104].

Although the specific pivotal mechanism(s) underlying this counter-regulation remains uncertain, one important contender is SOCS-1, which is T2 cytokine inducible and is a potent negative regulator of virus-induced IFN production (figure 1), partly at least through inhibition of Janus-activated kinase and STAT signalling, although the final mechanism of IFN inhibition requires further investigation $[44,105]$. Clearly this and other potential, pivotal, T2-T1 counter-regulation mechanisms require further exploration.

Additionally, in experimental chronic obstructive pulmonary disease (COPD) in mice, influenza worsened airway inflammation, and anti-IL-13 mAb improved this inflammation and IFN responses and reduced viral loads [41]. The potential dual mechanism of action hypothesis may therefore also apply to the use of T2 inhibitor therapies in patients with eosinophilic COPD, where at least one trial with mepolizumab (anti-IL-5 mAb) has shown a significant reduction in exacerbations in eosinophilic (i.e. blood eosinophils $\geqslant 150 \mathrm{~mL}^{-3}$ at screening or $\geqslant 300 \mathrm{~mL}^{-3}$ during the previous year) COPD patients [106]. Specifically, however, one T2 inhibitor, the GATA3-specific DNAzyme SB010, both attenuated sputum eosinophilia and resulted in a significant increase in blood IFN- $\gamma$ in eosinophilic COPD patients, supporting a similar dual mechanism of action of T2 inhibitors as seen in eosinophilic asthma described in this paper [107]. However, this latter study was relatively small and early, and further pre-clinical and clinical studies are required to confirm the potential dual mechanism of action and clinical benefit of T2 inhibitors in COPD. 
Although further clinical study validation is required, there already appears to be substantial pre-clinical and some clinical scientific support for a T2-T1 counter-regulation relevant to T2 inhibitor therapies in asthma, resulting from downregulation of T2 immune pathways leading to an upregulation of T1 immune pathways, including an increased IFN response. The inverse correlations between these T2 and T1 pathways and antiviral IFN responses, however, do not prove a causal link, although the relationship is clearly compelling and worthy of further exploration. The interaction between these related pathways may represent a form of "beneficial escape" mechanism, which in this case results in a potentially dual or additive clinical benefit. Clinical failure of other anti-inflammatory drugs tested in severe asthma, and indeed in nonrespiratory inflammatory disorders, however, may result from nonbeneficial escape or counter-regulation, in which one pro-inflammatory pathway is inhibited, but another interconnected pro-inflammatory pathway is consequently upregulated, negating or reducing any potential clinical benefit [108].

It seems probable, therefore, that the net positive and negative escape or counter-regulation mechanisms activated will also determine the overall clinical benefit and safety of any particular anti-inflammatory drug. This balancing of effects in an interconnected biological system may simply reflect on the fundamental and unifying regulatory mechanisms, first proposed by Claude Bernard, aimed at maintaining a constant or balanced internal environment ("milieu intérieur") following a significant perturbation [109].

It must be added that inhibiting any of the T2 mechanisms of asthma described here could reduce virally induced exacerbations independent of an effect on antiviral immunity by modifying at least one of these two additive and key inflammatory processes. However, reducing virally induced airway inflammation in asthma through both these mechanisms suggests an added and complementary potential benefit with T2 inhibitors, and may help to explain the substantial effect in reducing asthma exacerbations on top of corticosteroids already observed with a number of the anti-T2 mAbs.

\section{Conclusions}

Further basic science and clinical research to establish precisely how T2 cytokines can impair virus-induced IFN responses and vice versa is important to undertake, as the mechanisms involved could provide important novel therapeutic targets, as well as helping to better understand the mechanisms of action and clinical benefits of T2 and T1 targeted therapies, particularly in patients with virally induced asthma exacerbations. There also remains some uncertainty regarding the translatability of the results in murine models in this area, which as always require further confirmation in clinical studies. In addition, considering the growing wealth of clinical studies evaluating T2 biological and small-molecule inhibitors, the aforementioned dual mechanism hypothesis is clinically testable, and future exacerbation trials in the field and experimental viral challenge model should specifically examine the effects of T2 inhibitors on viral and other respiratory infections, viral clearance, and IFN responses, as well as on the treatment and prevention of virally induced exacerbations, in patients with asthma and COPD.

Author contributions: J. Efthimiou conceived the idea of the review and prepared the first draft of the manuscript, and C. Poll and P.J. Barnes both reviewed and revised the first draft. J. Efthimiou, C. Poll and P.J. Barnes reviewed and revised all subsequent drafts, including the submission draft that all authors approved. All three authors reviewed the literature searches, contents, analysis and interpretation of included papers and data. The figure was created by J. Efthimiou, and then reviewed and approved by all authors. No medical writers were employed to write this review.

Conflict of interest: J. Efthimiou has nothing to disclose. C. Poll has nothing to disclose. P.J. Barnes reports personal fees for advisory board work from AstraZeneca, grants and personal fees for advisory board work from Boehringer Ingelheim, and personal fees for lecturing from Novartis and Chiesi, during the conduct of the review, but not directly related to the scope of the review.

\section{References}

1 Johnston SL, Pattemore PK, Sanderson G, et al. Community study of role of viral infections in exacerbations of asthma in 9-11 year old children. BMJ 1995; 310: 1225-1229.

2 Atmar RL, Guy E, Guntupalli KK, et al. Respiratory tract viral infections in inner-city asthmatic adults. Arch Intern Med 1998; 158: 2453-2459.

3 Johnston SL, Pattemore PK, Sanderson G, et al. The relationship between upper respiratory infections and hospital admissions for asthma: a time-trend analysis. Am J Respir Crit Care Med 1996; 154: 654-660.

4 Grissell TV, Powell H, Shafren DR, et al. Interleukin-10 gene expression in acute virus-induced asthma. Am J Respir Crit Care Med 2005; 172: 433-439.

5 Busse WW, Lemanske RF, Gern JE. The role of viral respiratory infections in asthma and asthma exacerbations. Lancet 2010; 376: 826-834.

6 Kurai D, Saraya T, Ishii H, et al. Virus-induced exacerbations in asthma and COPD. Front Microbiol 2013; 4: 1-10.

7 Reddel H, Ware S, Marks G, et al. Differences between asthma exacerbations and poor asthma control. Lancet 1999; 353: 364-369. 
Oliver BGG, Robinson P, Peters M, et al. Viral infections and asthma: an inflammatory interface? Eur Respir J 2014; 44: 1666-1681.

Humbert M, Busse W, Hanania NA, et al. Omalizumab in asthma: an update on recent developments. J Allergy Clin Immunol Pract 2014; 2: 525-536.

Busse WW, Morgan WJ, Gergen PJ, et al. Randomized trial of omalizumab (anti-IgE) for asthma in inner-city children. N Engl J Med 2011; 364: 1005-1015.

Haldar P, Brightling CE, Hargadon B, et al. Mepolizumab and exacerbations of refractory eosinophilic asthma. N Engl J Med 2009; 360: 973-984.

Pavord ID, Korn S, Howarth P, et al. Mepolizumab for severe eosinophilic asthma (DREAM): a multicentre, double-blind, placebo-controlled trial. Lancet 2012; 380: 651-659.

Castro M, Zangrilli J, Wechsler ME, et al. Reslizumab for inadequately controlled asthma with elevated blood eosinophil counts: results from two multicentre, parallel, double-blind, randomised, placebo-controlled, phase 3 trials. Lancet Respir Med 2015; 3: 355-366.

Bleeker ER, FitzGerald JM, Chanez P, et al. Efficacy and safety of benralizumab for patients with severe asthma uncontrolled with high-dosage inhaled corticosteroids and long-acting $\beta_{2}$-agonists (SIROCCO): a randomised, multicentre, placebo-controlled phase 3 trial. Lancet 2016; 388: 2115-2127.

FitzGerald JM, Bleecker ER, Nair P, et al. Benralizumab, an anti-interleukin-5 receptor $\alpha$ monoclonal antibody, as add-on treatment for patients with severe, uncontrolled, eosinophilic asthma (CALIMA): a randomised, double-blind, placebo-controlled phase 3 trial. Lancet 2016; 388: 2128-2141.

Hanania NA, Noonan M, Corren J, et al. Lebrikizumab in moderate-to-severe asthma: pooled data from two randomised placebo-controlled studies. Thorax 2015; 70: 748-756.

Wenzel S, Ford L, Pearlman D, et al. Dupilumab in persistent asthma with elevated eosinophil levels. $N$ Engl Med 2013; 368: 2455-2466.

Wenzel S, Castro M, Corren J, et al. Dupilumab efficacy and safety in adults with uncontrolled persistent asthma despite use of medium-to-high-dose inhaled corticosteroids plus a long-acting $\beta_{2}$ agonist: a randomised double-blind placebo-controlled pivotal phase $2 \mathrm{~b}$ dose-ranging trial. Lancet 2016; 388: 31-44.

Corren J, Castro M, Jayawardena S, et al. Dupilumab improves asthma control and asthma-related quality of life in uncontrolled persistent asthma patients across all baseline exacerbation rates. Chest 2017; 152: Suppl., A26.

Castro M, Corren J, Pavord ID, et al. Dupilumab efficacy and safety in moderate-to-severe uncontrolled asthma. N Engl J Med 2018; 378: 2486-2496.

Barnes PJ. Cytokine inhibitors in the treatment of asthma and chronic obstructive pulmonary disease. Nat Rev Immunol 2018; 18: 454-466.

Fahy J. Type 2 inflammation in asthma - present in most, absent in many. Nat Rev Immunol 2015; 15: 57-65. Esquivel A, Busse WW, Calatroni A, et al. Effects of omalizumab on rhinovirus infections, illnesses and exacerbations of asthma. Am J Respir Crit Care Med 2017; 196: 985-992.

Teach SJ, Gill MA, Togias A, et al. Preseasonal treatment with either omalizumab or an inhaled corticosteroid boost to prevent fall asthma exacerbations. J Allergy Clin Immunol 2015; 136: 1476-1485.

Gill MA, Bajwa G, George TA, et al. Counter-regulation between the FceRI pathway and antiviral responses in human plasmacytoid dendritic cells. J Immunol 2010; 184: 5999-6006.

Durrani SR, Montville DJ, Pratt AS, et al. Innate immune responses to rhinovirus are reduced by the high-affinity IgE receptor in allergic asthmatic children. J Allergy Clin Immunol 2012; 130: 489-495.

Wark PA, Johnston SL, Bucchieri F, et al. Asthmatic bronchial epithelial cells have a deficient innate immune response to infection with rhinovirus. J Exp Med 2005; 201: 937-947.

Contoli M, Message SD, Laza-Stanca V, et al. Role of deficient type III interferon-lambda production in asthma exacerbations. Nat Med 2006; 12: 1023-1026.

Sykes A, Edwards MR, Macintyre J, et al. Rhinovirus 16-induced IFN-alpha and IFN-beta are deficient in bronchoalveolar lavage cells in asthmatic patients. J Allergy Clin Immunol 2012; 129: 1506-1514. an altered microRNA profile. Am J Respir Crit Care Med 2016; 194: 26-37.

Corren J, Parnes JR, Wang L, et al. Tezepelumab in adults with uncontrolled asthma. N Engl J Med 2017; 377: 936-946.

Salter BM, Oliveria JP, Nusca G, et al. IL-25 and IL-33 induce Type 2 inflammation in basophils from subjects with allergic asthma. Respir Res 2016; 17: 5.

Bateman ED, Guerreros AG, Brockhaus F, et al. Fevipiprant, an oral prostaglandin $\mathrm{DP}_{2}$ receptor (CRTh2) antagonist, in allergic asthma uncontrolled on low-dose inhaled corticosteroids. Eur Respir J 2017; 50: 1700670.

Steinke JW, Borish L. Th2 cytokines and asthma. Interleukin-4: its role in the pathogenesis of asthma, and targeting it for asthma treatment with interleukin-4 receptor antagonists. Respir Res 2001; 2: 66-70.

Gour N, Wills-Karp M. IL-4 and IL-13 signalling in allergic airway disease. Cytokine 2015; 75: 68-78.

Saatian B, Rezaee F, Desando S, et al. Interleukin-4 and interleukin-13 cause barrier dysfunction in human airway epithelial cells. J Tissue Barriers 2013; 1: e24333.

Baraldo S, Contoli M, Bazzan E, et al. Deficient antiviral immune responses in childhood: distinct roles of atopy and asthma. J Allergy Clin Immunol 2012; 130: 1307-1314.

Contoli M, Ito K, Padovani A, et al. Th2 cytokines impair innate immune responses to rhinovirus in respiratory epithelial cells. Allergy 2015; 70: 910-920.

Moran TM, Isobe H, Fernandez-Sesma A, et al. Interleukin-4 causes delayed virus clearance in influenza virus-infected mice. J Virol 1996; 70: 5230-5235.

Fischer JE, Johnson JE, Kuli-Zade RK, et al. Overexpression of interleukin-4 delays virus clearance in mice infected with respiratory syncytial virus. J Virol 1997; 71: 8672-8677.

Starkey MR, Dua K, Hsu AC, et al. Interleukin-13 predisposes to more severe influenza infection in mice and human epithelial cells by suppressing interferon responses and activating the microRNA-21/PI3K signalling pathway. Am J Respir Crit Care Med 2017; 195: A4926.

Hanania NA, Korenblat P, Chapman KR, et al. Efficacy and safety of lebrikizumab in patients with uncontrolled asthma (LAVOLTA I and LAVOLTA II): replicate, phase 3, randomised, double-blind, placebo-controlled trials. Lancet Respir Med 2016; 4: 781-796. 

STRATOS 2): two randomised, double-blind, placebo-controlled, phase 3 clinical trials. Lancet 2018; 6: 511-525.

44 Tabata Y, Khurana Hershey GK. IL-13 receptor isoforms: breaking through the complexity. Curr Allergy Asthma Rep 2007; 7: 338-345.

45 Wills-Karp M, Finkelman FD. Untangling the complex web of IL-4- and IL-13-mediated signalling pathways. Sci Signal 2008; 1: 55.

46 Wijesundara DK, Tscharke DC, Jackson RJ, et al. Reduced interleukin-4 receptor $\alpha$ expression on CD8 ${ }^{+} \mathrm{T}$ cells correlates with higher quality anti-viral immunity. PLoS One 2013; 8: e55788.

47 Albanesi C, Fairchild HR, Madonna S, et al. IL-4 and IL-13 negatively regulate TNF- $\alpha$ and IFN- $\gamma$ induced defensin expression through STAT-6 suppressor of cytokine signalling (SOCS)-1, and SOCS-3. J Immunol 2007; 179: 984-992.

48 Hammad H, Lambrecht BN. Dendritic cells and epithelial cells: linking innate and adaptive immunity in asthma. Nat Rev Immunol 2008; 8: 193-204.

49 Sanofi. FDA approves asthma indication for Dupixent (dupilumab). 2019. https://newsroom.regeneron.com/ news-releases/news-release-details/fda-approves-asthma-indication-dupixentr-dupilumab Date last accessed: April 12, 2019.

50 Fleming P, Drucker AM. Risk of infection in patients with atopic dermatitis treated with dupilumab: a meta-analysis of randomized controlled trials. J Am Acad Dermatol 2018; 78: 62-69.

51 Possa SS, Leick EA, Prado CM, et al. Eosinophilic inflammation in allergic asthma. Front Pharmacol $2013 ; 4: 46$.

52 Rosenberg HF, Dyer KD, Foster PS. Eosinophils: changing perspectives in health and disease. Nat Rev Immunol 2013; 13: 9-22.

53 Malik A, Batra JK. Antimicrobial activity of human eosinophil granule proteins: involvement in host defence against pathogens. Crit Rev Microbiol 2012; 38: 168-181.

54 Rosenburg HF, Dyer KD, Domachowske JB. Eosinophils and their interactions with respiratory virus pathogens. Immunol Res 2009; 43: 128-137.

55 Phipps S, Lam CE, Mahalingam S, et al. Eosinophils contribute to innate antiviral immunity and promote clearance of respiratory syncytial virus. Blood 2007; 110: 1578-1586.

56 Adamko DJ, Yost BL, Gleich GJ, et al. Ovalbumin sensitization changes the inflammatory response to subsequent parainfluenza infection. Eosinophils mediate airway hyper-responsiveness, $\mathrm{M}_{2}$ muscarinic receptor dysfunction, and antiviral effects. J Exp Med 1999; 190: 1465-1478.

57 Drake MG, Bivins-Smith ER, Proskocil BJ, et al. Human and mouse eosinophils have antiviral activity against parainfluenza virus. Am J Respir Cell Mol Biol 2016; 55: 387-394.

58 Domachowske JB, Dyer KD, Adams AG, et al. Eosinophil cationic protein/RNase 3 is another RNase A-family ribonuclease with direct antiviral activity. Nucleic Acids Res 1998; 26: 3358-3363.

59 Domachowske JB, Dyer KD, Bonville CA, et al. Recombinant human eosinophil-derived neurotoxin/RNase 2 functions as an effective antiviral agent against respiratory syncytial virus. J Infect Dis 1998; 177: 1458-1464.

60 Hatchwell L, Collison A, Girkin J, et al. Toll-like receptor 7 governs interferon and inflammatory responses to rhinovirus and is suppressed by IL-5 induced lung eosinophilia. Thorax 2015; 70: 845-846.

61 Jackson DJ, Makrinioti H, Rana BM, et al. IL-33-dependent type 2 inflammation during rhinovirus-induced asthma exacerbations in vivo. Am J Respir Crit Care Med 2014; 190: 1373-1382.

62 Efthimiou J, Mann A, Balaratnam G, et al. Airway inflammation and clinical manifestations in patients with mild asthma: time course of human rhinovirus (HRV) induced changes. Am J Respir Crit Care Med 2016; 193 : A1429.

63 Mann A, Ghebre MA, Poll CT, et al. Predictors of human rhinovirus induced asthma disease: clinical, cellular and biomarker predictors at baseline and change post infection in the human challenge model. Am J Respir Crit Care Med 2018; 197: A7170.

64 Pineros YSS, Bal SM, van de Pol MA, et al. Anti-IL5 in mild asthma alters rhinovirus-induced macrophage, B cell and neutrophil responses (MATERIAL): a placebo-controlled, double-blind study. Am J Respir Crit Care Med 2019; 199: 508-517.

65 Sy CB, Siracusa MC. The therapeutic potential of targeting cytokine alarmins to treat allergic airway inflammation. Front Physiol 2016; 7: 214.

66 Phipps S, Werder R, Zhang V, et al. Persistent IL-33 in a preclinical chronic asthma model underpins rhinovirus-induced exacerbation by dampening antiviral immunity. Am J Respir Crit Care Med 2017; 195 : A6994.

67 Ravanetti L, Dijkhaus A, Dekker T, et al. IL-33 drives influenza-induced asthma exacerbations by halting innate and adaptive antiviral immunity. J Allergy Clin Immunol 2019; 143: 1355-1370.

68 Werder RB, Zhang V, Lynch JP, et al. Chronic IL-33 expression predisposes to virus-induced asthma exacerbations by increasing type 2 inflammation and dampening antiviral immunity. J Allergy Clin Immunol 2018; 141: 1607-1619.

69 Regeneron. Study of safety, tolerability, and pharmacokinetics of multiple ascending doses of REGN3500 in adults with moderate asthma. 2018. https://clinicaltrials.gov/ct2/show/NCT02999711 Date last accessed: April 12, 2019.

70 AnaptysBio. Proof of concept study to investigate ANB020 activity in adult patients with severe eosinophilic asthma. 2018. https://clinicaltrials.gov/ct2/show/NCT03469934 Date last accessed: April 12, 2019.

71 GSK. Efficacy and safety study of GSK3772847 in subjects with moderately severe asthma. 2017. https:// clinicaltrials.gov/ct2/show/NCT03207243 Date last accessed: April 12, 2019.

72 Kato A, Favoreto S Jr, Avila PC, et al. TLR3- and Th2 cytokine-dependent production of thymic stromal lymphopoietin in human airway epithelial cells. J Immunol 2007; 179: 1080-1087.

73 Boita M, Bucca C, Riva G, et al. Release of type 2 cytokines by epithelial cells of nasal polyps. J Immunol Res 2016; 2016: 2643297.

74 Nino G, Huseni S, Perez GF, et al. Directional secretory response of double stranded RNA-induced thymic stromal lymphopoetin (TSLP) and CCL11/eotaxin-1 in human asthmatic airways. PLoS One 2014; 9: e115398.

75 Bjerregaard A, Laing IA, Poulsen N, et al. Characteristics associated with clinical severity and inflammatory phenotype of naturally occurring virus induced exacerbations of asthma in adults. Respir Med 2017; 123 : 344.

76 Mahmutovic-Persson I, Akbarshahi H, Bartlett NW, et al. Inhaled dsRNA and rhinovirus evoke neutrophilic exacerbation and lung expression of thymic stromal lymphopoietin in allergic mice with established experimental asthma. Allergy 2014; 69: 348-358. 
Stier MT, Bloodworth MH, Toki S, et al. Respiratory syncytial virus infection activates IL-13-producing group 2 innate lymphoid cells through thymic stromal lymphopoietin. J Allergy Clin Immunol 2016; 138: 814-824. blood and airway gene expression in patients with persistent eosinophilic asthma. Am J Respir Crit Care Med 2018; 197: A6164.

79 Novartis. Study of efficacy and safety of QAW039 in patients with severe asthma inadequately controlled with standard of care asthma treatment. 2015. https:/clinicaltrials.gov/ct2/show/NCT02555683 Date last accessed: April 12, 2019.

80 Barnes N, Pavord I, Chuchalin A, et al. A randomized, double-blind, placebo-controlled study of the CRTH2 antagonist OC000459 in moderate persistent asthma. Clin Exp Allergy 2012; 42: 38-48.

81 Imperial College London. Effect of OC459 on the response to rhinovirus challenge in asthma. 2015. https:// clinicaltrials.gov/ct2/show/NCT02660489 Date last accessed: April 12, 2019.

82 Moore WC, Bleecker ER, Curran-Everett D, et al. Characterization of the severe asthma phenotype by the National Heart, Lung, and Blood Institute's Severe Asthma Research Program. J Allergy Clin Immunol 2007; 119: 405-413.

83 Smith JR, Noble MJ, Musgrave S, et al. The at-risk registers in severe asthma (ARRISA) study: a cluster-randomised controlled trial examining effectiveness and costs in primary care. Thorax 2012; 67: 1052-1060.

84 Jackson DJ, Johnston SL. The role of viruses in acute exacerbations of asthma. J Allergy Clin Immunol 2010; 125: $1178-1187$.

85 Israel E, Reddel HK. Severe and difficult to treat asthma in adults. N Engl J Med 2017; 377: 965-976.

86 Miller MK, Lee JH, Miller DP, et al. Recent asthma exacerbations: a key predictor of future exacerbations. Respir Med 2007; 101: 481-489.

87 Krishnan V, Diette GB, Rand CS, et al. Mortality in patients hospitalized for asthma exacerbations in the United States. Am J Respir Crit Care Med 2006; 174: 633-638.

88 Dougherty RH, Fahy JV. Acute exacerbations of asthma: epidemiology, biology and the exacerbation-prone phenotype. Clin Exp Allergy 2009; 39: 193-202.

89 Venarske DL, Busse WW, Griffin MR, et al. The relationship of rhinovirus-associated asthma hospitalizations with inhaled corticosteroids and smoking. J Infect Dis 2006; 193: 1536-1543.

90 Djukanovic R, Harrison T, Johnston SL, et al. The effect of inhaled IFN- $\beta$ on worsening of asthma symptoms caused by viral infections. Am J Respir Crit Care Med 2014; 190: 145-154.

91 Synairgen. AZD9412 Update. 2017. https://uk.advfn.com/stock-market/london/synairgen-SNG/share-news/ Synairgen-plc-AZD9412-Update/74433922 Date last accessed: April 12, 2019

92 Becker Y. The changes in the T helper 1 (Th1) and T helper 2 (Th2) cytokine balance during HIV-1 infection are indicative of an allergic response to viral proteins that may be reversed by Th2 cytokine inhibitors and immune response modifier: a review and hypothesis. Virus Genes 2004; 28: 5-18.

93 Allen JE, Sutherland TE, Ruckerl D. IL-17 and neutrophils: unexpected players in the type 2 immune response. Curr Opin Immunol 2015; 34: 99-106.

94 Castro M, Chaplin DD, Walter MJ, et al. Could asthma be worsened by stimulating the T-helper type 1 immune response? Am J Respir Cell Mol Biol 2000; 22: 143-146.

95 Cohn L, Tepper JS, Bottomly K. IL-4-independent induction of airway hyperresponsiveness by Th2, but not Th1, cells. J Immunol 1998; 161: 3813-3816.

96 Ying S, Durham SR, Corrigan CJ, et al. Phenotype of cells expressing mRNA for TH2-type (interleukin 4 and interleukin 5) and TH1-type (interleukin 2 and interferon $\gamma$ ) cytokines in bronchoalveolar lavage and bronchial biopsies from atopic asthmatic and normal control subjects. Am J Respir Cell Mol Biol 1995; 12: 477-487.

97 Swain SL, Bradley LM, Croft M, et al. Helper T-cell subsets: phenotype, function and the role of lymphokines in regulating their development. Immunol Rev 1991; 123: 115-144.

98 Seder RA, Paul WE. Acquisition of lymphokine producing phenotype by CD41 T cells. Ann Rev Immunol 1994; 12: 635-637.

99 Edwards MR, Strong K, Cameron A, et al. Viral infections in allergy and immunology: how allergic inflammation influences viral infections and illness. J Allergy Clin Immunol 2017; 140: 909-920.

100 Huber JP, Ramos HJ, Gill MA, et al. Cutting edge: type I IFN reverses human Th2 commitment and stability by suppressing GATA3. J Immunol 2010; 185: 813-817.

101 Edwards S, Jones C, Leishman AJ, et al. TLR7 Stimulation of APCs results in inhibition of IL-5 through type I IFN and Notch signalling pathways in human peripheral blood mononuclear cells. J Immunol 2013; 190: 2585-2592.

102 Subrata LS, Bizzintino J, Mamessier E, et al. Interactions between innate antiviral and atopic immunoinflammatory pathways precipitate and sustain asthma exacerbations in children. J Immunol 2009; 183: 2793-2800.

103 Duerr CU, McCarthy CD, Mindt BC, et al. Type I interferon restricts type 2 immunopathology through the regulation of group 2 innate lymphoid cells. Nat Immunol 2015; 17: 65-75.

104 Koltsida O, Hausding M, Stavropoulos A, et al. IL-28A (IFNג2) modulates lung DC function to promote Th1 immune skewing and suppress allergic airway disease. EMBO Mol Med 2011; 3: 348-361.

105 Gielen V, Sykes A, Zhu J, et al. Increased nuclear suppressor of cytokine signalling 1 in asthmatic bronchial epithelium suppresses rhinovirus induction of innate interferons. J Allergy Clin Immunol 2015; 136: 177-188.

106 Pavord ID, Chanez P, Criner GJ, et al. Mepolizumab for eosinophilic chronic obstructive pulmonary disease. $N$ Engl J Med 2017; 377: 1613-1629.

107 Greulich T, Hohlfeld JM, Neuser P, et al. A GATA3-specific DNAzyme attenuates sputum eosinophilia in eosinophilic COPD patients: a feasibility randomized clinical trial. Respir Res 2018; 19: 55-64.

108 Brightling C, Berry M, Amrani Y. Targeting TNF-alpha: a novel therapeutic approach for asthma. J Allergy Clin Immunol 2008; 121: 5-10.

109 Bernard C. Lecons sur les Phenomenes de la Vie Communes aux Animaux et aux Vegetaux [1878; Reprint]. Paris, J. Vrin, 1966 